\title{
An Object-Based Method for Rician Noise Estimation in MR Images
}

\author{
Pierrick Coupé ${ }^{1}$, José V. Manjón ${ }^{2}$, Elias Gedamu ${ }^{1}$, Douglas Arnold ${ }^{1}$, \\ Montserrat Robles ${ }^{2}$, and D. Louis Collins ${ }^{1}$
}

1 McConnell Brain Imaging Centre, Montréal Neurological Institute, McGill University, 3801 University Street, Montréal, Canada H3A 2B4

2 Biomedical Informatics Group (IBIME), ITACA Institute, Universidad Politécnica de Valencia, Camino de Vera, s/n. 46022 Valencia, Spain

\begin{abstract}
The estimation of the noise level in MR images is used to assess the consistency of statistical analysis or as an input parameter in some image processing techniques. Most of the existing Rician noise estimation methods are based on background statistics, and as such are sensitive to ghosting artifacts. In this paper, a new object-based method is proposed. This method is based on the adaptation of the Median Absolute Deviation (MAD) estimator in the wavelet domain for Rician noise. The adaptation for Rician noise is performed by using only the wavelet coefficients corresponding to the object and by correcting the estimation with an iterative scheme based on the SNR of the image. A quantitative validation on synthetic phantom with artefacts is presented and a new validation framework is proposed to perform quantitative validation on real data. The results show the accuracy and the robustness of the proposed method.
\end{abstract}

\section{Introduction}

In MR image analysis, the estimation of the noise level in an image is a mandatory step that must be addressed to assess the quality of the analysis and the consistency of the image processing technique. The noise variance is also an important measure for many image processing techniques such as denoising [1,2] or registration. Furthermore, procedures that employ statistical analysis techniques, such as functional MR imaging or voxel-based morphometry, often base their conclusions on assumptions about the underlying noise characteristics. Usually, the real and imaginary parts of the MR complex raw data are considered corrupted by white additive Gaussian noise, where the noise variance is assumed to be the same in both parts (real and imaginary) [3, 4]. By taking the magnitude of the complex data, the noise is transformed into Rician noise $[3,4,5,6,7$, . Conventionally, the Rician noise is $i$ ) described by a Rayleigh distribution in the background [3,4,6, 7] (i.e. the signal of air in the background is considered to be zero), and ii) approximated by Gaussian noise in the foreground when Signal Noise Ratio (SNR) is high enough $(>3 d B$ [1]). These models for background and foreground noise distribution have been used in the majority of noise estimation methods $[8,6,7$. However, the Rayleigh model of the background can fail 
when ghosting artefacts are present (i.e. non-zero signal) [6], and the Gaussian approximation of foreground is no longer valid for images with low SNR 6]. Some automatic techniques have been proposed [8, 6, 7. Usually, these methods use the histogram of the background and some properties of the Rayleigh distribution. Recently, a new noise Rician variance estimation method based on maximum likelihood (ML) estimation from a partial histogram was presented by Sijbers 6. More recently, Aja et al. 7] presented a set of new methods for noise estimation based on local statistics. In this paper, an adaptation of the Median Absolute Deviation (MAD) estimator in the wavelet domain is proposed for Rician noise. This robust and efficient estimator has been proposed by Donoho [9] for Gaussian noise and since has been widely used in image processing. We propose to adapt this operator for Rician noise by using only the wavelet coefficients corresponding to the object and then iteratively correcting the MAD estimation with an analytical scheme based on the SNR of the image [5].

\section{Noise in MR Images}

As mentioned previously, the distribution of noise can be modeled with a Rician distribution [3, 4, 6, 10,

$$
p(m)=\frac{m}{\sigma_{n}^{2}} \exp \left(-\frac{m^{2}+A^{2}}{2 \sigma_{n}^{2}}\right) I_{0}\left(\frac{A m}{\sigma_{n}^{2}}\right) .
$$

where $\sigma_{n}$ is the standard deviation of Gaussian noise in the complex domain, $A$ is the amplitude of the signal without noise, $m$ is the value in the magnitude image and $I_{0}$ is the zeroth order modified Bessel function. This model is used by the majority of the noise estimation methods [8,6, 7,. Most of these methods can be classified as: $i$ ) methods that use background areas to estimate the noise variance and $i i$ ) methods that use the image object itself.

- For the background-based methods, where the signal is usually considered as zero in background (i.e. $\mathrm{SNR}=0 d B$ ), the Rician distribution is a Rayleigh distribution [6]:

$$
p(m)=\frac{m}{\sigma_{n}^{2}} \exp \left(-\frac{m^{2}}{2 \sigma_{n}^{2}}\right) .
$$

Based on the properties of the Rayleigh distribution, the mean $\bar{m}_{b}$ and the variance $\sigma_{b}^{2}$ of the noise in the background can be related to $\sigma_{n}$ :

$$
\begin{gathered}
\bar{m}_{b}=\sqrt{\frac{\pi}{2}} \sigma_{n} \\
\sigma_{b}^{2}=\frac{4-\pi}{2} \sigma_{n}^{2}
\end{gathered}
$$

The assumption that $\mathrm{SNR}=0 d B$ in the background may not be valid in the presence of ghosting artefacts [6], while the Rayleigh distribution assumption 
can be corrupted by using reconstruction filters [11,10], by the suppression of the signal by the scanner [10,11] or by zero-padding in the Fourier domain 11. Finally, the noise level in the background may not be representative of the noise level inside the tissue [10,11].

- For the object-based methods with high SNR (i.e. SNR > 3dB) 4], the Rician distribution can be well approximated using a Gaussian distribution:

$$
p(m) \approx \frac{1}{2 \pi \sigma_{n}^{2}} \exp \left(-\frac{\left(m^{2}-\sqrt{A^{2}+\sigma_{n}^{2}}\right)^{2}}{2 \sigma_{n}^{2}}\right) .
$$

This approximation enables us to use all the classical methods proposed for Gaussian noise estimation. Nevertheless, for low SNR, this approximation is no longer valid [4, 1, 6].

\section{Proposed Method}

In order to relax the assumptions performed by background-based methods (i.e. no signal in the background) and the object-based methods (Gaussian noise approximation), we propose an adaptation of the MAD estimator in wavelet domain 9] for Rician noise.

MAD Estimator. By using the usual notation for 3D wavelet decomposition: LLL denotes the low sub-band containing the feature information whereas LHH, LHL, LLH, HLL, HLH, HHL and HHH denote the high sub-bands containing the detailed information. The highest sub-band $\mathrm{HHH}$ is essentially composed of coefficients that correspond to the noise [9]. The fact that the highest sub-band $\mathrm{HHH}$ is mainly composed of the coefficients corresponding to the noise has been used by Donoho [9] to propose a robust estimation of noise variance. Based on the MAD estimator, this method enables the estimation of the noise variance in presence of Gaussian noise:

$$
\hat{\sigma}=\frac{\operatorname{median}\left(\left|y_{i}\right|\right)}{0.6745}
$$

where $y_{i}$ are the wavelet coefficients of the HHH sub-band and $\hat{\sigma}$ the estimation of noise. As long as the $y_{i}$ coefficients corresponding to the object are considered and the SNR is high enough, the Gaussian approximation of Rician noise leads to $\hat{\sigma}_{n}=\hat{\sigma}$.

Rician Adaptation. To obtain an unbiased estimation of $\sigma_{n}$ for all SNR values, we propose to use the correction procedure introduced by Koay et al [5]. This analytical correction is based on an iterative estimation of the SNR in presence of Rician noise. In our case, the estimation $\hat{\sigma}$, obtained using the MAD estimator on the object, is used to initialize the procedure:

$$
\hat{\sigma}_{n}=\sqrt{\hat{\sigma}^{2} / \xi(\theta)}
$$


where $\theta$ is the SNR value and $\xi$ is the correction factor, which is expressed as:

$$
\xi(\theta)=2+\theta^{2}-\frac{\pi}{8} \times \exp \left(-\frac{\theta^{2}}{2}\right)\left(\left(2+\theta^{2}\right) I_{0}\left(\frac{\theta^{2}}{4}\right)+\theta^{2} I_{1}\left(\frac{\theta^{2}}{4}\right)\right)^{2}
$$

where $I_{1}$ is the first order modified Bessel function. The correction factor is iteratively applied until convergence of the procedure or when a given number of iterations $t$ is achieved. The distance $\left|\theta_{t}-\theta_{t-1}\right|$ can be used as stopping criterion. The resulting iterative correction scheme can be written as:

$$
\theta_{t}=\sqrt{\xi\left(\theta_{t-1}\right)\left(1+\frac{\bar{m}_{o}}{\hat{\sigma}}\right)-2}
$$

where $\bar{m}_{o}$ is the mean signal of the object and $\hat{\sigma}$ the first estimation from MAD estimator. The correction factor $\xi\left(\theta_{t}\right)$ from the last iteration is finally used in Eq.7

Object Extraction. The first approximation, $\hat{\sigma}_{n} \approx \hat{\sigma}$, is solely based on the wavelet coefficients corresponding to the object. To extract the object we take advantage of the wavelet transform. Since the noise information is mainly contained in the highest sub-bands, the LLL sub-band contains a less noisy version of the image which can be used to facilitate the segmentation procedure. At the first level of decomposition, the size of LLL and HHH are identical. Thus, at this level of decomposition, we proposed to segment the object in the LLL sub-band and to use the obtained mask to extract the $y_{i}$ coefficients corresponding to the object in the HHH sub-band. The segmentation is performed using a simple K-means $(k=2)$ classification. For image with a low level of noise, the MAD estimation tends to be spoiled since the HHH sub-band is mainly composed of information corresponding to the high gradient areas (i.e. edges) of the image. To further increase the accuracy of the estimation at low noise levels, voxels with the highest local gradient are excluded from the estimation (i.e. removed from the segmented mask). Accordingly, we eliminate all those voxels whose the local gradient magnitude is higher than the median local gradient magnitude in the LLL sub-band.

\section{Experiment on Synthetic Data}

Materials. To evaluate the different methods, synthetic T1-weighted MR data with $20 \%$ of inhomogeneity from the Brainweb database [12] was corrupted with different levels of Rician noise (2 to $15 \%$ ). In this paper, $2 \%$ of noise is equivalent to $\mathcal{N}\left(0, \nu \frac{2}{100}\right)$, where $\nu$ is set to 255 . As shown in [7], the size of the background has an impact on the accuracy of the background-based methods. Smaller backgrounds lead to more difficult estimations. In order to perfrom a fair comparison, zero padding of the Brainweb volume of $181 \times 217 \times 181$ voxels was performed to obtain a volume of $256 \times 217 \times 256$ voxels. Moreover, ghosting artefacts were implemented by using a repeated filtered version of the original image. First, the image is low-pass filtered with two gaussian kernels of different size $(3 \times 3 \times 3$ and $5 \times 5 \times 5)$. Then, the absolute difference of the two filtered images is added to the original image with a half field of view offset (see Fig. 1 left). 

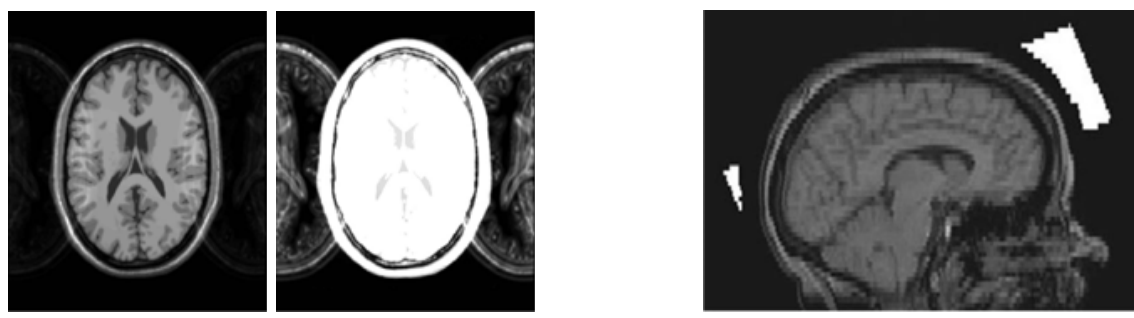

Fig. 1. Left: Simulated ghosting artefacts on brainweb with $20 \%$ inhomogeneity and the same image with saturation of the contrast to highlight the ghosting artefacts. Right: Noise regions of interest obtained with automated quality control software tool 13 .

Compared Methods. For the experiments, we compared the following methods:

- the background-based method proposed by Sijbers 6]; denoted as "ML". The Sijbers method was applied using a histogram with 1000 bins.

- the two background-based methods based on local statistics proposed in [7]; denoted as LMB for the Local Means in Background and LVB for Local Variances in Background. The size of the local neighborhoods and the number of bins were $5 \times 5 \times 5$ voxels and 1000 bins respectively.

- the object-based method based on local variances proposed in [7]; denoted as LVO for Local Variances in Object. A local neighborhood of $3 \times 3 \times 3$ voxels was used.

- the classical MAD estimator estimated on the object 9]. The object was segmented in the wavelet domain without removing high gradient areas.

- the proposed robust MAD for Rician noise estimated on the object and denoted as RMAD.

Quality Measure. To estimate the accuracy of the different methods, the ratio between the estimated standard deviation $\hat{\sigma}_{n}$ and the applied standard deviation $\sigma_{n}$ is computed for all the levels of noise. Moreover, the Mean Absolute Error over all the levels of noise is also used. The error for a given level of noise is computed as:

$$
\text { error }=1-\frac{\sigma_{n}}{\hat{\sigma_{n}}}
$$

All the experiments were repeated 10 times, each with a new instantiation of noise, for each noise level and the average results are presented.

Results. Fig. 2 shows the results on the phantom with inhomogeneity and ghosting artefacts. Compared to the MAD estimator, the ability of the RMAD method to correctly estimate the higher levels of noise (i.e. where the Gaussian assumption failed) can be attributed to the SNR based correction factor. Moreover, the RMAD provided better estimations of the noise at low level by removing the high gradients before the MAD computation. As expected, the background-based methods are impacted by the ghosting artefacts. In fact, the assumption of zero signal in 

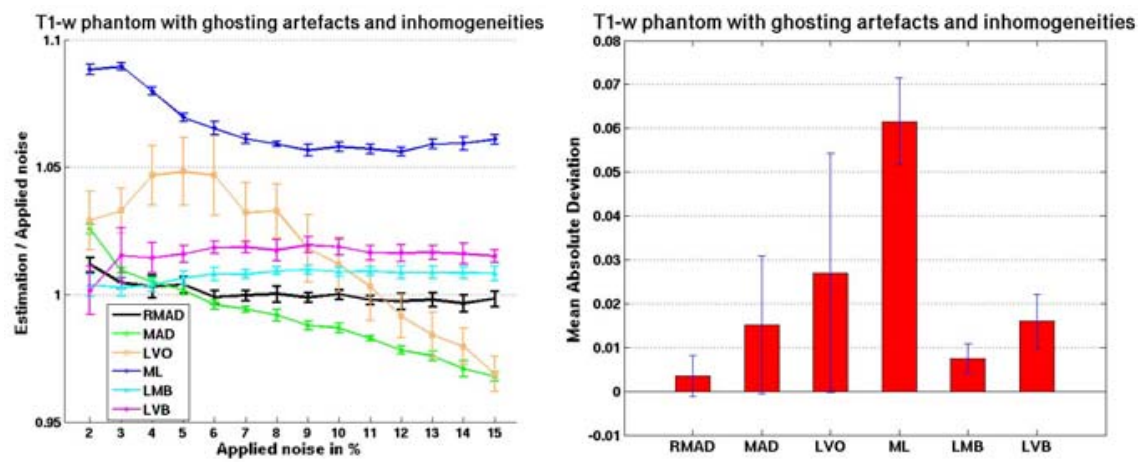

Fig. 2. Left: ratio of estimated vs. applied noise level, for the all noise levels applied to the synthetic image, for the 6 techniques compared. Note that RMAD (black line) estimation is closest to 1.0 for almost all noise levels.

the background is spoiled. All these methods tend to overestimate the noise level, especially the ML method. The LMB method obtained very good results. Finally, the RMAD method obtained the best result.

\section{Experiments on Clinical Data}

Material. The dataset used for the experiment is composed of 23 T1-w MR volumes of $256 \times 256 \times 56$ voxels. These data were acquired with a $1.5 \mathrm{~T}$ Genesis Signa GE Medical system and an 1 channel head coil. The parameters of the sequence were: $\mathrm{TR}=30 \mathrm{~ms}, \mathrm{TE}=9 \mathrm{~ms}, \mathrm{FOV}=250 \mathrm{~mm}$ and bandwidth $=122 \mathrm{kHz}$.

Background Extraction. In order to estimate the noise level in the real images, we used a region-based approach that is similar to the manual selection procedure usually used in the clinical environment. The noise region of interest (ROI) used to calculate the noise level was obtained by using the automated quality control (aQC) software tool described in [13. Based on the registration of each subject with a template of ROIs (see Fig. 1, right), the AQc software provides ROIs associated with noise regions. To determine the noise level, we used the region anterior to the head which contains less artefacts [13.

Bronze Standard. In our study, we have chosen to use the assumption that the noise level for a given sequence on the same scanner should be constant. Based on this idea, the noise regions extracted from the background of MR images are used to estimated an average level of noise over all the data from a same site. To estimate this average level of noise, the properties of the second-order moment of a Rician distribution are used. The Bronze standard can be computed from the mean of the squared values extracted from backgrounds of all the data $d$ :

$$
\hat{\sigma}_{n}=\sqrt{\frac{\overline{\hat{M}_{b}^{2}}}{2}}, \hat{M}_{b}^{2}=\left(\hat{m}_{b}^{2}(1), \ldots, \hat{m}_{b}^{2}(D)\right)
$$



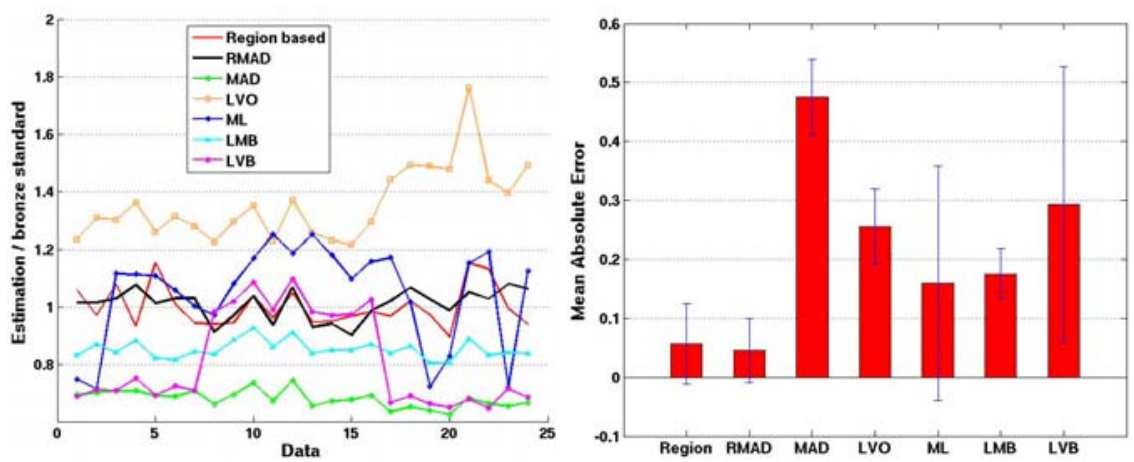

Fig. 3. Left: results of the compared methods for all the data. Right: mean absolute error over all the data.

where $\hat{m}_{b}^{2}(d)$ is the vector containing the squared value of noise extracted from the background of data $d$ and $\hat{M}_{b}^{2}$ is the concatenation of the squared signal from all the data. Based on the same approach, the estimation of the noise level for a given data $d$ is obtained with the region-based method by using the mean of the squared values extracted from the background of $d$.

Results. Fig. 3 shows the results obtained for site 1. For all the data, RMAD method provided a consistant estimation of the noise relative to the Bronze standard (small error) in a robust manner (small variance of error). The stability of the proposed method leads to a smaller error than the region-based method that was used to build the bronze standard. As assessed by experiments on synthetic phantom, the MAD estimator computed on the object tends to underestimate the noise level whereas the LVO method leads to an overestimation of the noise level. The LVB method appears to follow the MAD estimation and the RMAD estimation according to the data under process. The LMB method estimated in the background is a robust and stable estimator but leads to a slight underestimation. Finally, the ML method provided a good mean absolute error but was accomplished with a high variability.

\section{Conclusion}

In this paper, a new method based on the robust MAD estimator for Rician noise has been proposed and several state-of-the-art methods for Rician noise estimation in MR image have been compared. Experiments on synthetic data with simulated inhomogeneity and ghosting artefacts showed the efficienty of the proposed object-based approach compared to the background-based approaches. Experiments on real data have shown that the proposed RMAD method obtained the best result in terms of accuracy and robustness. The background-based methods had the highest variability except the LMB method which provided a robust noise estimation. These results show that the background in real data is spoiled by artefacts, thus violating the assumption of no signal in background. Finally, 
the proposed approach can be applied to situations where no background is present such as fetal imaging or images where the background is artificially set to zero by the scanner 10 . Moreover, the proposed approach can be potentially adapted to work with non stationary noise such as those attributed to parallel imaging (i.e. GRAPPA or SENSE).

Acknowledgments. We are grateful to Dr. Sijbers and Dr. Aja for providing the source code of their respective Rician noise estimation methods and for their useful comments. We want to thank Dr Levesque for the fruitfully discussions about MR image acquisition. We would like to thank NeuroRx Research for providing the data. This work has been partially supported by the Canadian Intitutes of Health Research Industry Cda (CECR)-Gevas-OE016 and by the Spanish Health Institute Carlos III through the RETICS Combiomed, RD07/0067/2001.

\section{References}

1. Nowak, R.: Wavelet-based rician noise removal for magnetic resonance imaging. IEEE TIP 8(10), 1408-1419 (1999)

2. Coupé, P., Yger, P., Prima, S., Hellier, P., Kervrann, C., Barillot, C.: An Optimized Blockwise NonLocal Means Denoising Filter for 3-D Magnetic Resonance Images. IEEE Transactions on Medical Imaging 27(4), 425-441 (2008)

3. Henkelman, R.M.: Measurement of signal intensities in the presence of noise in MR images. Medical Physics 12(2), 232-233 (1985)

4. Gudbjartsson, H., Patz, S.: The Rician distribution of noisy MRI data. Magnetic Resonance in Medicine 34, 910-914 (1995)

5. Koay, C.G., Basser, P.J.: Analytically exact correction scheme for signal extraction from noisy magnitude MR signals. Journal of Magnetic Resonance 179(2), 317-322 (2006)

6. Sijbers, J., Poot, D., den Dekker, A.J., Pintjens, W.: Automatic estimation of the noise variance from the histogram of a magnetic resonance image. Physics in Medicine and Biology 52(5), 1335-1348 (2007)

7. Aja-Fernandez, S., Alberola-Lopez, C., Westin, C.F.: Noise and signal estimation in magnitude MRI and Rician distributed images: a LMMSE approach. IEEE TIP 17(8), 1383-1398 (2008)

8. Sijbers, J., den Dekker, A., Audekerke, J.V., Verhoye, M., Dyck, D.V.: Estimation of the noise in magnitude MR images. Magnetic Resonance Imaging 16(1), 87-90 (1998)

9. Donoho, D.: De-noising by Soft-Thresholding. IEEE TIT 41(3), 613-627 (1995)

10. Dietrich, O., Raya, J.G., Reeder, S.B., Ingrisch, M., Reiser, M.F., Schoenberg, S.O.: Influence of multichannel combination, parallel imaging and other reconstruction techniques on MRI noise characteristics. Magnetic Resonance Imaging 26(6), 754 $762(2008)$

11. Landman, B., Bazin, P.L., Prince, J.: Diffusion Tensor Estimation by Maximizing Rician Likelihood. In: ICCV 2007, pp. 1-8 (2007)

12. Collins, D., Zijdenbos, A., Kollokian, V., Sled, J., Kabani, N., Holmes, C., Evans, A.: Design and construction of a realistic digital brain phantom. IEEE TMI 17(3), 463-468 (1998)

13. Gedamu, E.L., Collins, D.L., Arnold, D.L.: Automated quality control of brain MR images. Journal of Magnetic Resonance Imaging 28(2), 308-319 (2008) 